\title{
I sistemi informativi di supporto alle decisioni (DSS) nell'attivita' di pianificazione e valutazione ambientale degli impianti geotermici a bassa entalpia: attualita' e prospettive di sviluppo
}

\author{
Marco Bersano Begey \\ DHI-Italia, HYDRODATA, Torino \\ bersano.begey@dhi-italia.it
}

La diffusione esponenziale negli ultimi anni della tecnologia di riscaldamento e condizionamento con impianti geotermici a bassa entalpia, ha comportato la necessità di mettere a punto strumenti e tecnologie adeguate per la valutazione delle condizioni tecniche di gestione e degli impatti sull'ambiente idrico sotterraneo.

Infatti, il ricorso intensivo agli scambi di calore con l'acquifero, dove non correttamente gestito e pianificato può determinare effetti di rilevanza sia "ambientale", connessi alla diffusione del plume termico, sia sotto il profilo "energetico" (disponibilità e bilancio della risorsa).

Dal punto di vista ambientale gli effetti come noto non sono trascurabili, e sono potenzialmente dovuti a "derive" termiche di riscaldamento e raffreddamento dell'acquifero.

Dal punto di vista energetico, occorre considerare il calore geotermico come una risorsa rinnovabile ma non infinita, ovvero con precisi limiti di sfruttamento, evitando sovrautilizzi non compatibili con l'equilibrio termico dell'acquifero, tali da rendere inefficienti, e impattanti sotto il profilo ambientale, impianti concepiti e progettati per operare in condizioni diverse e più favorevoli.

In pratica occorre guardare al bilancio energetico della risorsa geotermica esattamente nei medesimi termini con cui si analizza il bilancio di fluido nell'acquifero, dove i prelievi idrici eccedenti la capacità di ricarica influiscono sul cono di depressione piezometrico, ed i prelievi di calore sul profilo delle temperature nella fascia di profondità interessata dagli scambiatori o dai pozzi di reimmissione.

Inoltre, anche l'inserimento di nuovi prelievi da falda nell'ambito di settori interessati da impianti a ciclo aperto può determinare modificazioni piezometriche locali e indurre condizioni di corto-circuito termico, con perdita di funzionalità di impianti esistenti.

E' evidente dunque, che la pianificazione della risorsa in contesti complessi, caratterizzati da una sovrapposizione di prelievi sotterranei di fluido e di energia, non può essere effettuata senza l'utilizzo di strumenti, di modellistica concettuale e numerica, che consentano di valutare l'inserimento e le modalità di utilizzo di ogni impianto nel contesto dell'effettiva disponibilità della risorsa, e della compatibilità con i prelievi di energia già in essere.

Tale attività di valutazione non può essere effettuata solo dal progettista, alla scala del singolo impianto, ma deve essere necessariamente riferita a sistemi complessi su area vasta, e affrontata sulla base di metodologie, tecniche, e assunzioni relative all'ambiente fisico di riferimento in assoluto omogenee.
In settori di sfruttamento intensivo della risorsa idrica sotterranea, come alcune aree metropolitane, l'approccio attuale, sia in ambito nazionale che internazionale, si basa sulla costruzione di Sistemi informativi di Supporto alle Decisioni (DSS), in grado di costituire riferimento per i progettisti nella fase propositiva e per gli Enti di controllo nella fase autorizzativa.

Un DSS, anche nel campo dell'analisi di matrici ambientali, può essere efficamente descritto dalla definizione generale, con minimi adattamenti, che ne dà l'inesauribile Wikipedia:

"Un Decision Support System (DSS) è un sistema informativo di supporto alle decisioni, che permette di aumentare l'efficacia dell'analisi in quanto fornisce supporto a tutti coloro che devono prendere decisioni strategiche di fronte a problemi che non possono essere risolti con i modelli della ricerca operativa. La funzione principale di un DSS è quella di estrarre in poco tempo e in modo versatile le informazioni utili ai processi decisionali, provenienti da una rilevante quantità di dati.

Il DSS si appoggia su dati in un database o una base di conoscenza, che aiutano l'utilizzatore a decidere meglio; esso non è solo un'applicazione informatica, perché contiene anche strumenti di Business Intelligence e di tecnologie dei Sistemi Esperti, quali modelli di supporto decisionale."

Nell'ambito delle risorse idriche l'esempio pilota più rappresentativo è quello dell'Area Metropolitana di Milano, in cui l'equilibrio delle falde con i prelievi rappresenta da sempre un aspetto di particolare criticità, e dove il gestore del Servizio Idrico Integrato (Metropolitana Milanese S.p.A.) si è da tempo dotato di un sistema $D S S$, costruito sulla base del codice numerico avanzato MIKE-SHE (DHI), affidandone la successiva gestione tecnica e l'aggiornamento alla Provincia di Milano (Zelioli et al., 2009).

Tale sistema è costituito da un modello numerico del ciclo delle acque superficiali-sotterranee, comprensivo della zona non satura, calibrato in regime transitorio sulla base di oltre un decennio di dati idrologici a passo giornaliero. Attraverso tale strumento, è possibile simulare efficacemente e in tempi rapidi qualsiasi variabile al contorno del sistema (es. nuovi prelievi, impermeabilizzazione delle reti di canali, variazioni di utilizzo del suolo di rilevanza sui parametri di infiltrazione/evapotraspirazione, andamenti idrologici particolari del regime di ricarica, ecc.).

Il DSS, che interessa un esteso settore tra Adda e Ticino, è stato utilizzato recentemente per la valutazione rapida di scenari relativi a grandi progetti di approvvigionamento idrico, sia per uso potabile sia per eventuale scambio termico, con particolare riferimento al progetto Expo 2015. 
Il sistema è stato successivamente esteso anche alle simulazioni di trasporto, limitatamente ai composti dell'azoto, e consente di valutare a supporto dei processi decisionali gli effetti relativi a interventi di riduzione del carico in acque superficiali e sotterranee.

La costruzione di DSS estesi alla valutazione della risorsa geotermica e dell'impatto di grandi prelievi di energia è un approccio in progressiva diffusione in ambito internazionale. A riguardo un esempio di alto livello tecnico-scientifico è il sistema di valutazione messo a punto a partire dal 2009 da London Transport, in riferimento all'area centrale urbana di Londra, nell'ambito del progetto "Cooling the Tube Programme" (Arthur e Heather Streetly, 2009). In tale ambito un sistema di modellizzazione dell'intera area urbana di Londra è stato messo a punto per la valutazione degli effetti termici conseguenti al progetto di climatizzazione delle gallerie e stazioni della rete metropolitana sotterranea, mediante impianti geotermici open-loop.

Il modello di larga scala, riaggiornato, costituisce un sistema informativo DSS efficace per l'Ente pubblico di supporto alle decisioni.

Sul territorio nazionale, pur non essendo stati portati a un livello operativo e trasferiti all'ente pubblico o al gestore DSS in ambito geotermico, sono in fase realizzativa da parte di DHI-Italia con HYDRODATA S.p.A. sistemi sperimentali in ambiente FEFLOW, per le aree metropolitane di Milano (sulla base del DSS - Flow-Transport già operativo) e di Torino, al fine di testare lo strumento di calcolo su applicazioni che per scala territoriale risultano particolarmente impegnative dal punto di vista del calcolo numerico.

A riguardo, anticipando aspetti che potranno essere affrontati in altra sede più ampia, lo sviluppo del DSS si basa su un "motore" ("engine") costituito dall'accoppiamento di un modello idrologico superficiale con un codice di simulazione del flusso saturo / non saturo e trasporto di energia (Diersch et al, 2009, Konzen, 2011).

In ambito commerciale sono a riguardo disponibili sistemi accoppiati in ambiente DHI MIKE11 / FEFLOW, DHI MIKE11/ MODFLOW-MT3D+SEAWAT, per citare i codici più diffusi.

Rimandando a sede più ampia il confronto tra i codici di calcolo numerico attualmente disponibili, le attività in corso per la realizzazione dei DSS a livello sperimentale, come precedentemente citato, vengono sviluppate per il comparto sotterraneo in ambiente FEFLOW.

Utilizzando tale codice gli impianti esistenti e in progetto sono inseriti nel modello sia sulla base di approcci estremamente dettagliati, dove note le complete modalità operative e la struttura funzionale, sia in forma semplificata, attraverso l'inserimento della potenza scambiata in $\mathrm{kW}$ ai nodi di modello in corrispondenza di uno scambiatore di calore.

Questo approccio "semplificato" può essere estremamente utile per l'inserimento, in particolare, degli impianti a scambiatore in pozzo $(B H E)$ a circuito chiuso, spesso di potenza limitata ma diffusi sul territorio, che costituiscono dunque una condizione al contorno importante per la valutazione di inserimento dei più potenti impianti a ciclo aperto.

E' pertanto possibile implementare il modello anche in condizioni locali di scarsa o incompleta disponibilità di dati, assumendo input, per gli impianti minori, anche solo correlabili alla domanda di energia termica presunta in funzione della dimensione e tipologia media degli edifici.

Per gli impianti a ciclo aperto, è necessario che il codice implementi una funzionalità per imporre un differenziale $\Delta \mathrm{T}$ di temperatura o l'equivalente $\Delta \mathrm{P}$ di potenza termica tra acque emunte e reimmesse in falda. Tale funzionalità consente di valutare gli effetti degli impianti sulla temperatura della falda in presenza di un'energia scambiata pari alla potenza nominale di ogni impianto ovvero al fabbisogno termico (es. su base mensile o media stagionale) degli edifici serviti. La medesima funzionalità consente inoltre di valutare condizioni di corto circuito termico tra pozzi di iniezione e di reimmissione, anche indotti dalla deviazione delle linee di flusso a seguito di pompaggi limitrofi in condizioni di interferenza.

Il modello con implementati i dati relativi agli impianti esistenti, costituisce dunque il DSS di livello "0", ovvero il sistema in grado di descrivere lo stato attuale degli impatti e gli scenari di deriva termica futuri nell'ipotesi del mantenimento nel tempo della medesima condizione di riferimento.

Il DSS può a questo punto essere utilizzato per la valutazione di inserimento di nuovi impianti e la pianificazione di utilizzo della risorsa.

In questo senso, svolgendo un ruolo di riferimento per la pianificazione, ad un livello superiore a quello della progettualità locale, il DSS deve necessariamente essere costruito attraverso passi costantemente condivisi con l'Ente pubblico preposto alla valutazione (ARPA, Provincie), e dopo la validazione essere trasferito all'Ente pubblico medesimo affidandone, quale strumento di pubblica utilità, gestione e aggiornamento.

\section{BIBLIOGRAFIA}

Arthur S., Heather Streetly H. (2009) Modelling of groundwater cooling schemes in London. International FEFLOW conference, 2009.

Zelioli A., Marelli F., Cargnelutti M. (2009) Studio e modellazione del flusso idrico nel sistema acquifero del territorio milanese. Italian DHI Conference. Torino, 6-7 ottobre 2009.

Diersch J.G., Rühaak W., Schätzl P., Renz A. (2009) A New Method for Modelling Geothermal Heat Exchangers in Shallow Aquifer Systems. International FEFLOW conference, 2009.

Konzen, Thomas (2011) Energetische Grundwassernutzung im städtischen Bereich - Planung bei konkurrierenden Nutzungen. Bbr Fachmagazin für Brunnen- und Leitungsbau. 